

Abstract PTU-122 Figure 1 Early vs late unplanned admissions

highest risk. However, more studies are needed to see if these results are reproducible and related to individual procedure. Early endoscopy readmissions could therefore be used as a surrogate marker for endoscopic complications and has the potential to be used in a KPI study.

\section{PTU-123 ENDORINGS ASSISTED COLONOSCOPY VERSUS STANDARD COLONOSCOPY FOR POLYP DETECTION: A RANDOMISED CONTROLLED TRIAL}

${ }^{1}$ Sreedhari Thayalasekaran*, ${ }^{1}$ Rupam Bhattacharyya, ${ }^{2}$ Fergus Chedgy, ${ }^{3}$ Peter Basford, ${ }^{1}$ Asma Alkandari, 'Sharmila Subramaniam, 'Kesavan Kandiah, 'Fergus Thursby-Pelham, ${ }^{1}$ Richard Ellis, 'Sergio Coda, 'Patrick Goggin, 'Gaius Longcroft-Wheaton, ${ }^{1}$ Pradeep Bhandari. ${ }^{1}$ Queen Alexandra Hospital, Portsmouth, UK; ${ }^{2}$ Royal Sussex County Hospital, Brighton, UK; ${ }^{3}$ St Richards Hospital, Chichester, UK

\subsection{6/gutjnl-2019-BSGAbstracts.112}

Introduction The Endorings ${ }^{\mathrm{TM}}$ is a distal attachment consisting of two layers of circular flexible rings that evert mucosal folds. Aims; to investigate if Endorings Colonoscopy (ER) improves polyp and adenoma detection compared to standard colonoscopy (SC).

Methods This multi-centre, parallel group, randomized controlled trial included screening, surveillance and symptomatic patients. Primary outcome; number of polyps per patient. Secondary outcomes; number of adenomas per patient, adenoma/ polyp detection rates and withdrawal times.

Results Total of 556 patients (214 females, 342 males) randomized to ER (275) or SC (281). Mean age 67. Colonoscopy completed 532/556 (96\%) cases. EndoRings removed in $74 / 275(27 \%)$ patients. In $66 / 74(89 \%)$ cases removal was performed due to difficulties with sigmoid intubation. Remainder removed to facilitate retroflexion or polyp removal/retrieval. Total number of polyps in ER limb 571 vs 444 in SC limb. Total number of adenomas in ER limb 361 vs 343 for SC limb. Our study showed a statistically significant difference in the mean number of polyps per patient in both the Intention to Treat (ITT) (1.8 SC vs $2.1 \mathrm{ER}$, p-value $0.02)$ and Per Protocol (PP) (1.8 SC vs 2.25 ER, p-value $0.009)$.

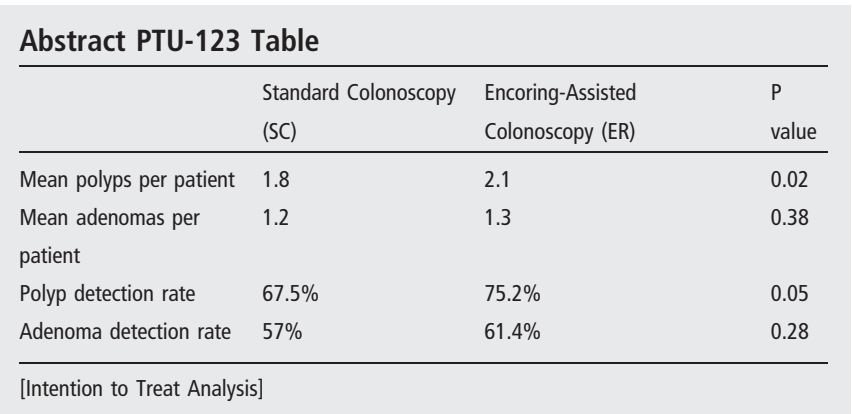

There was a trend towards a greater polyp detection rate in the ER colonoscopy $(67.5 \%$ SC vs $75.2 \%$ ER, p-value $0.05)$.

Conclusions Despite the high removal rate of Endorings, there was a statistically significant increase in the mean number of polyps in the ER limb compared to the SC limb. Our study shows promise for the EndoRings ${ }^{\mathrm{TM}}$ device to improve polyp detection.

\section{PTU-124 INCREASED DIAGNOSTIC SENSITIVITY OF PANCREATICOBILIAY MALIGNANCY BY MODIFYING ERCP BRUSHING PRACTICE: A SINGLE CENTRE EXPERIENCE}

Ioannis Varmpompitis*, Khansa Adam, Jonathan Booth, Sinead McEvoy, Nishchay Chandra. Royal Berkshire Hospital, Nea Smirni, Athens, Greece

10.1136/gutjnl-2019-BSGAbstracts.113 
Introduction Brushings at ERCP play a crucial role in establishing a diagnosis in cases of biliary strictures. In this study, we examined if brushing practice can make a difference to the diagnostic yield resulting in less interventions. We analysed ERCP data over the last two and a half years at the Royal Berkshire Hospital, where inclusion of the brush head for cytology and performing three or more passages across the stricture has been standard practice.

Methods We looked at results of brushings taken by 2 ERCPists in a single centre between September 2016 to February 2018. The initial brushing result from each individual patient was included. Brushing results were classified as non-diagnostic, negative, atypical, suspicious or positive for malignancy. Brushings classified as suspicious and identified as malignant were categorised into the positive group; those classified as non-diagnositc, non-malignant and atypical were categorised as negative. For comparison purposes we considered the corresponding histology and/or radiological findings and/or positive immunohistochemistry in the follow-up period. Data was analysed using the statistical package SPSS v.25.

Results In total, 71 individual brushing outcomes were identified over a 2.5-year period. In 61 of 71 cases $(85.9 \%)$ the final diagnosis was cancer, with the majority being pancreatic cancer $(36 / 61 ; 59 \%)$. The sensitivity was estimated at $77 \%$ (47/61 true positive), the specificity and the positive predictive value (PPV) were both $100 \% \quad(0$ false negative, 10 true negative), while the negative predictive value (NPV) was $38 \%$. When we looked at only 'positive for malignancy' results, the sensitivity stood at $60 \%$. In $15 / 61$ cases $(24.6 \%)$ a supplementary report was needed to confirm the result and it changed the outcome in only 3 cases (20\%).

Conclusions Biliary brushings are a very useful means of providing a diagnosis during ERCP and are characterised by a high PPV and low NPV. Negative results should not be interpreted as absence of malignancy but if the yield is at least suspicious, the specificity approaches 100\%. This study suggests that sending the brush head for cytology and performing three or more passages across the stricture increases diagnostic sensitivity when compared to published data reporting on sensitivities up to 64\% (Burnett AS, Calvert TJ, Chokshi RJ. Sensitivity of endoscopic retrograde cholangiopancreatography standard cytology: 10-y review of the literature. J Surg Res. 2013;184:304-11.)

\section{PTU-125 FACTORS INFLUENCING INCREASED ERCP BRUSHING SENSITIVIY IN PANCREATICOBILIARY MALIGNANCY: A SINGLE CENTRE EXPERIENCE}

loannis Varmpompitis*, Cameron Griffiths, Sinead McEvoy, Jonathan Booth, Nishchay Chandra. Royal Berkshire Hospital, Reading, UK

\subsection{6/gutjnl-2019-BSGAbstracts.114}

Introduction Biliary brushings are often the only way to confirm a diagnosis of malignancy in patients presenting with biliary strictures. There is a paucity of data regarding the parameters that may affect the diagnostic yield of this technique. The aim of this study was to identify key factors that may improve diagnostic sensitivity in hepatobiliary malignancies.

Methods Brushing outcomes were identified over a 2-year period and analysed by demographic factors (age, sex), indication of the procedure, site and length of stenosis on ERCP, CBD dilatation on CT/MRCP/ERCP, site and size of mass, laboratory values (ALP, ALT, bilirubin) prior to the procedure, and final outcome (histologically or radiologically confirmed). We calculated the ratio of at least suspicious for malignancy results out of the total cases of malignancies. Final outcome was defined by histology or radiological evidence of cancer in the follow-up period. Data was retrospectively retrieved and processed using the EPR (electronic patient record) hospital database and the radiology InSight PACS system. Data was analysed using the statistical package SPSS v.25.

Results A total of 59 brushing results were identified over the period 01/2017-01/2019. $52(88.1 \%)$ were malignant and 7 (11.9\%) were benign. The mean age was 71.4 yrs and the majority of malignancies were pancreatic cancer (33/52; $63.5 \%$ ), with the remainder including cholangiocarcinoma (7), ampullary cancer (6) and other malignancies. Forty of 52 cases were true positives, indicating a sensitivity of $76.9 \%$, while the specificity was $100 \%$ (no false negative and $7 / 7$ true negative).

Among the factors examined, sensitivity was significantly associated with the site of stenosis $(89.2 \%$ for distal vs. $50 \%$ for mid and mid-distal stenoses, $\mathrm{p}=0.023$ ), and with the mass being in the pancreatic head versus in the uncinate process (84.6 vs $40 \%, p=0.029)$. Sensitivity was higher for longer strictures $(63.6 \%$ vs $46 \%$ for strictures $>1.9 \mathrm{~cm}$ (i.e. the median value), and for larger masses (mean size of mass $4 \mathrm{~cm}$ in true positive vs $2.6 \mathrm{~cm}$ in false negatives) but these results did not reach statistical significance. The age, CBD dilatation on imaging, type of cancer and the laboratory markers before the procedure were not associated with differences in the diagnostic yield.

Conclusions Distal CBD stenoses and pancreatic head lesions are associated with statistically significant increase in brushing sensitivity for malignancy. This study suggests that in the absence of a distal CBD stricture or a pancreatic head lesion, further investigation modalities (additional brushings, EUS, percutaneous biopsy, cholangioscopy) will be more likely required to achieve diagnostic certainty.

\section{PTU-126 RESULTS OF USING COLORECTAL SEMS VS DECOMPRESSION TUBE AS BRIDGE TO SURGERY: COMPARATIVE RANDOMIZED TRAIL}

${ }^{1,2,3}$ Alexandr Vodoleev*, ${ }^{1}$ Vladimir Duvanskiy, ${ }^{2}$ Sergey Pirogov, ${ }^{3}$ Denis Kryazhev, ${ }^{2}$ lliya Perfilev, ${ }^{2}$ Dmitriy Sukhin, ${ }^{2}$ Elena Karpova, ${ }^{2}$ Andrey Kniazev, ${ }^{1}$ Ivan Yarotskov. ${ }^{1} R U D N$, Moscow, Russian Federation; ${ }^{2}$ National Medical Research Center of Radiology, Moscow, Russia; ${ }^{3}$ Eramishantsev Clinical Hospital, Moscow, Russia

\subsection{6/gutjnl-2019-BSGAbstracts. 115}

Introduction Despite advances in screening and early diagnosis, about $15 \%$ of colorectal cancers present with acute colonic 